\title{
Material
}

\section{Analysis of Variance in Large Bowel Cancer Patients within a Clinical Pathway Using POSSUM}

\author{
Naokuni YASUdA ${ }^{1)}$, Makoto W $_{\text {ATANABE }}{ }^{2)}$, Osamu NAKashima ${ }^{1)}$ \\ Toru OHNAKA ${ }^{1)}$, Akira TsunOda ${ }^{2)}$ and Mitsuo Kusano ${ }^{2)}$
}

\begin{abstract}
The aim of this study was to assess analysis of variance of clinical pathways for large bowel cancer patients using the scoring system physiological and operative severity score for the enumeration of mortality and morbidity (POSSUM), which predicts postoperative morbidity and mortality. Large bowel cancer cases performed in our department between January 2002 and March 2004 were studied. These were divided into three groups : group one consisted of 48 patients without variance; group two consisted of 13 patients without variance and with complications; and group three consisted of 13 patients with variance. The average postoperative complication risk predicted by POSSUM was $34.4 \%$. Observed complications were recorded in 26 out of 74 patients $(35.1 \%)$, and variance developed in 13 of these 26 patients. There were no differences in the PS and OSS scores between patients with and without variance. Predicting the occurrence of variance using POSSUM is difficult as many factors can influence the development of variance. Therefore, POSSUM is unlikely to be a suitable scoring system for predicting which patients will develop variance.
\end{abstract}

Key words : POSSUM, clinical pathway, variance, colorectal cancer

\section{Introduction}

A clinical pathway is essential for efficient outcome management within the medical service $^{1)}$. The length of hospital stay and criteria for patient discharge are the two crucial outcomes. Variances are patient outcomes or staff actions that do not meet the expectations of a clinical pathway ${ }^{2)}$. Reasons for variances are generally categorized as being patient-related, staff-related, hospital-related and social factors. Most patient-related factors of variances are due to postoperative complications or a poor understanding of discharge guidelines.

Many scoring systems predict the risk of mortality and morbidity with varying degrees of accuracy ${ }^{3)}$. The physiological and operative severity score for the enumeration of mortality and morbidity (POSSUM) system was first described by Copeland et $a l^{4)}$ in 1991. Twelve physiological and six operative factors were developed for scoring, and these scores were applied to an equation to obtain a predicted postoperative morbidity rate for each patient. POSSUM was found to be the most accurate predictive system compared to the other available scoring systems ${ }^{5}$.

\footnotetext{
1) Department of Surgery, Isesaki Municipal Hospital, 12-1 Tsunatorihonmachi, Isesaki 372-0817, Japan.

${ }^{2)}$ Second Department of Surgery, Showa University, School of Medicine.
} 
The aim of this study was to apply POSSUM analysis to assess the variance of clinical pathways for large bowel cancer patients.

\section{Materials and Methods}

Patients who underwent elective colorectal resection (excluding planned abdominoperineal examination of the rectum) between January 2002 and March 2004 as part of a clinical pathway were selected for this study. Patients whose performance status was rated at less than 2 on the POSSUM system were excluded, because palliative operations were sometimes undertaken instead of resection surgery. The remaining patients were divided into three groups: patients without variance or complications; patients without variance and with complications; and patients with variance.

Implementation of a clinical pathway in our department is characterized by patient education and clear discharge criteria. At the induction of a clinical pathway and prior to surgery, we outlined six goals that should be achieved before discharge to the patients and their families ${ }^{6,7)}$. These goals included progression through the following phases: pain, effort, temperature, meals, shower and examination. These goals were achieved when the patient was comfortable with or without oral painkiller, could walk independently, ate solid meals more than $50 \%$ of the time, maintained a normal temperature for 48 hours, was able to take a shower, and had examination results that fell within the normal range before discharge.

The clinical pathway applied to the patients is described below and is based on the ability of patients to achieve the six goals before discharge by postoperative day 14 . On the morning of postoperative day 1 , the nasogastric tube was removed. On postoperative day 3, the epidural and Foley catheters were removed, and the patient was started on sips of clear liquid. On postoperative day 5, the patient was allowed a liquid diet. For the purpose of this study, variance was defined as when a patient was not able to achieve the six goals by postoperative day 16 and therefore not discharged.

The POSSUM system is based on scores from a four-grade, 12-factor physiological score (PS) and a six-factor operative severity score (OSS), which estimates the risk of adverse postoperative outcome (Table 1). The risk was calculated from logistic regression equations $^{4,5)}$. The equation used for postoperative morbidity ( $R$ : Outcome Risk) was: $\operatorname{In}[\mathrm{R} /(1-\mathrm{R})]=-5.9+(0.16 \times \mathrm{PS})+(0.19 \times \mathrm{OSS})$

\section{Statistical analysis}

Differeces between observed and predicted outcomes were assessed using chi-squared test and the Hosmer-Lemeshow goodness-of-fit test. The statistical significance of differences between patients with variance and patients without variance and complications was tested by student's t-test; a $\mathbf{P}$ value of less than 0.05 was considered significant.

\section{Results}

A total of 74 patients were involved in this study. These included 57 colic resections ( 31 right-sided hemicolectomies and 26 sigmoid resections or left-sided hemicolectomies) and 17 anterior resections or ultra-low anterior resections. Postoperative complications were observed in 26 patients $(35.1 \%), 13$ of whom developed variance. Table 2 shows characteristics of patients without variance and complications, patients without variance and 


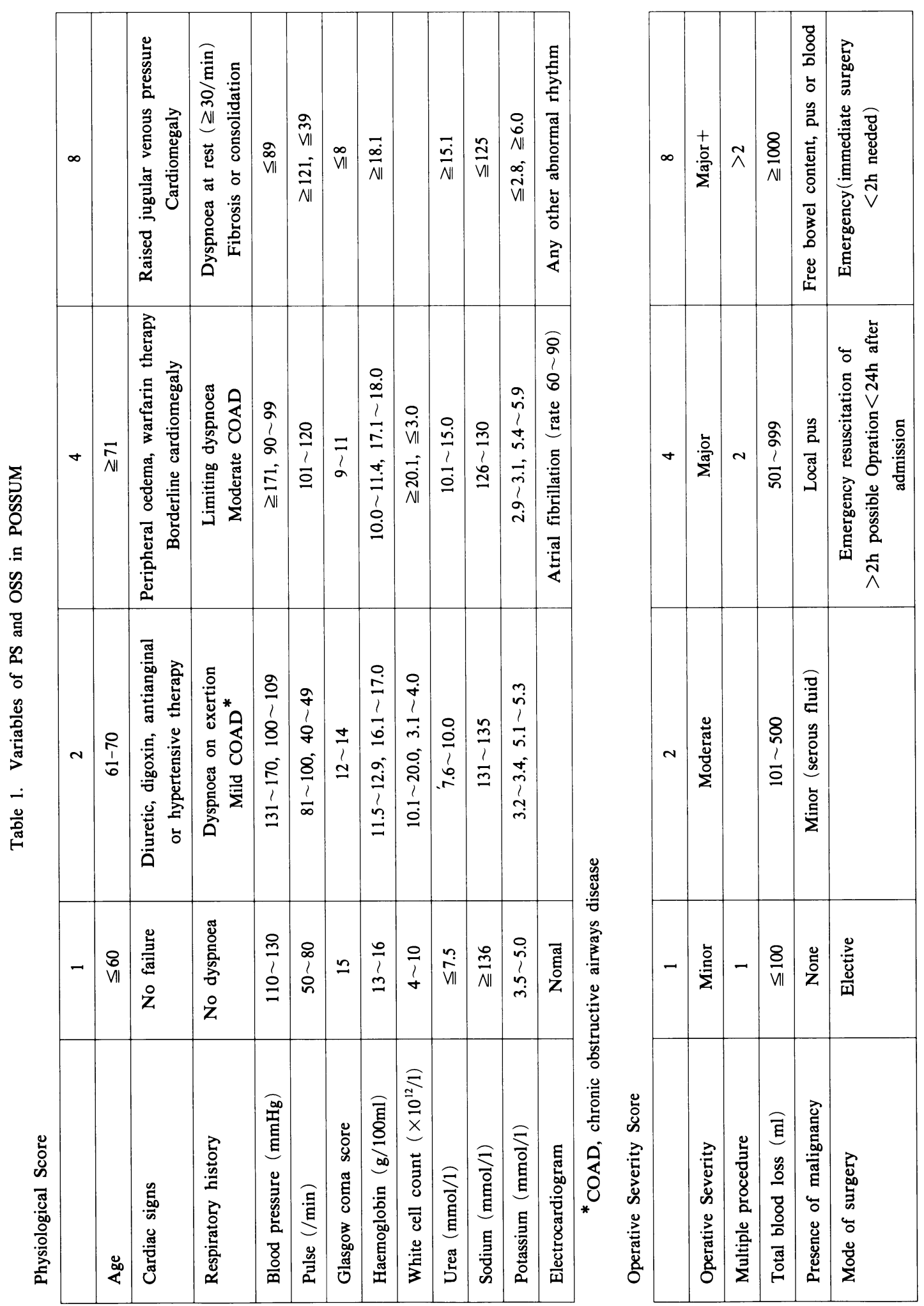


Table 2. Clinical characteristics of patients without valiance and complications, patients without valiance and with complications, patients with valiance

\begin{tabular}{|c|c|c|c|}
\hline & \multicolumn{2}{|c|}{ without variance } & \multirow[t]{2}{*}{ with variance } \\
\hline & $\begin{array}{c}\text { without } \\
\text { complications }\end{array}$ & $\begin{array}{c}\text { with } \\
\text { complications }\end{array}$ & \\
\hline No. of cases & 48 & 13 & 13 \\
\hline Age & $63 \pm 9.9$ & $68.9 \pm 11.3$ & $64.2 \pm 13.3$ \\
\hline Sex & $28: 20$ & $10: 3$ & $6: 7$ \\
\hline \multicolumn{4}{|l|}{ Location } \\
\hline Right sided & 18 & 5 & 8 \\
\hline Left sided & 20 & 3 & 3 \\
\hline Rectum & 10 & 5 & 2 \\
\hline \multicolumn{4}{|l|}{ Stage } \\
\hline 0 & 0 & 1 & 1 \\
\hline I & 7 & 2 & 0 \\
\hline II & 21 & 7 & 6 \\
\hline III $\mathbf{A}$ & 11 & 1 & 5 \\
\hline III B & 3 & 0 & 0 \\
\hline IV & 6 & 2 & 1 \\
\hline
\end{tabular}

Table 3. Comparison of complication rate between POSSM and observed

\begin{tabular}{cccccc}
\hline & \multicolumn{2}{c}{ Hosmer-Lemeshow goodness-of-fit test for the POSSUM morbidity equation } \\
\hline & & \multicolumn{2}{c}{ Complication $(-)$} & \multicolumn{2}{c}{ Complication $(+)$} \\
\hline Predicted rete $(\%)$ & No. of patients & Observed & Predicted & Observed & Predicted \\
\hline $9.0 \sim 12.7$ & 8 & 6 & 8.1 & 2 & 0.9 \\
\hline $14.9 \sim 17.9$ & 7 & 7 & 5.8 & 0 & 1.2 \\
\hline $18.5 \sim 19.9$ & 9 & 6 & 7.3 & 3 & 1.7 \\
\hline $20.4 \sim 24.4$ & 7 & 5 & 5.4 & 2 & 3 \\
\hline $25.0 \sim 30.6$ & 7 & 4 & 5.1 & 4 & 2.8 \\
\hline $30.8 \sim 38.0$ & 8 & 4 & 5.2 & 5 & 2.9 \\
\hline $38.5 \sim 44.8$ & 7 & 2 & 4.1 & 2 & 3.2 \\
\hline $45.5 \sim 47.3$ & 7 & 5 & 3.8 & 3 & 3.7 \\
\hline $47.8 \sim 58.4$ & 7 & 4 & 3.3 & 1 & \\
\hline $61.8 \sim 97.3$ & 7 & 6 & 1.5 & $\chi^{2}=27.1,8$ d.f., p=0.00066
\end{tabular}

with complications, and patients with variance.

The mean complication rate predicted by POSSUM was $34.4 \%$, which was comparable with the observed morbidity rate of $35.1 \%$. In patients with observed morbidity, surgical site infection (SSI) was seen in $73.1 \%$, ileus in $11.5 \%$, delirium in $11.5 \%$, and leakage in 7.7\%. There was, however, no relationship between the rate predicted by POSSUM and 
Table 4. Comparison of POSSUM results between patients without valiance and complications and patients with valiance

\begin{tabular}{lccc}
\hline & $\begin{array}{c}\text { without variance } \\
\text { and complications }\end{array}$ & with variance & p-value \\
\hline No. of cases & 48 & 13 & \\
PS & $17.1 \pm 4.4$ & $17.1 \pm 4.3$ & 0.9963 \\
OSS & $12.9 \pm 4.3$ & $12.9 \pm 2.8$ & 0.9828 \\
POSSUM & $34.2 \%$ & $35.4 \%$ & 0.8593 \\
\hline
\end{tabular}

observations using the Hosmer-Lemeshow goodness-of-fit test (Table 3). The group with the highest predicted rete consisted of two patients who had undergone ultra-low anterior resection and four patients who had undergone colectomy (mean PS $=23$, range 14-31; mean OSS $=20$, range $13-29$ ). In the patients with variance, SSI was seen in $69.2 \%$, ileus in $23.1 \%$, and leakage in $15.4 \%$. There were no differences in PS, OSS and the rate predicted by POSSUM between patients with variance and patients without variance and complications (Table 4).

\section{Discussion}

Clinical pathways have varying formats and are known by many names, including critical pathways and care paths ${ }^{8}$. Clinical pathways were originally adopted from engineering fields. First developed in the 1950 s, the Critical Path Method and Program Evaluation and Review technique were used in the industrial setting to manage the efficiency and cost effectiveness of projects ${ }^{9,10}$. Clinical pathways were first developed and applied to health care in the $1980 \mathrm{~s}$, when economic pressure and cost-effective medical practices developed in the United States ${ }^{8,11,12)}$. Therefore, the goal of clinical pathways was to provide high quality care in a cost-effective manner ${ }^{8,13,14}$. In recent years, clinical pathways have become common in all areas of Japanese medicine. Clinical pathways encompass preoperative and intraoperative patient care in addition to postoperative care. Rather than simply reducing the length of hospital stay and health-care costs, clinical pathways are also useful for improving staff coordination, patient satisfaction, and patient care ${ }^{7,15}$.

Clinical pathways are advantageous to the safe management of medicine as they inform patients of the standard treatment schedule. The quality of surgical care influences morbidity, mortality and length of hospital stay. Previous studies ${ }^{16-18)}$ have found that clinical pathways can lead to a decrease in the cost and length of hospital stay without increasing morbidity and mortality in bowel resection procedures. However, these studies were not prospective randomized controlled studies. Such studies are difficult because a single institute needs a large number of patients and it is possible that increased surgical experience will contribute to a decrease in complications over time.

Recently, Haga et al $^{19,20)}$ suggested that a scoring system used to predict postoperative risk might be useful for surgical decision making, medical expense estimations, and comparisons of surgical quality. Theoretically, if the number of variables is smaller, then the scoring system will be more user-friendly ${ }^{7)}$. POSSUM is a widely used scoring system that predicts postoperative risk of mortality and morbidity. The 12 PS factors are assessed at admission as part of the routine clinical history and examination, which includes biochemical, 
radiological and cardiological investigations. The six features of OSS are assessed postoperatively. As POSSUM is based on both pre-and post-surgical criteria, we chose POSSUM to predict variance in patienes within a clinical pathway.

In the present study, there was no relationship between the observed incidence of variance and risk predicted by POSSUM, although patient-related postoperative complications developed in all patients with variance. Our results suggested that the most significant difference was observed in the group with the highest predicted rate, between patients without complications and patients with complications. A possible explanation is that our medical care combined with a clinical pathway is effective for patients, especially those at high-risk. Therefore, a clinical pathway including patient education, in combination with good medical care, may help prevent variance in patients that have operable large bowel cancers.

Our results showed that there were no correlations between the complication rate predicted by POSSUM and the observed complication rate. Although some studies ${ }^{21,22)}$ have suggested that POSSUM should be modified according to ethnicity or disease, we used the original POSSUM in this study. Due to the small number of patients in this study, we are unable to comment on the validity of these modifications. Further studies are required to determine whether these modifications should be incorporated. However, such predictive scores might be useful to inform and educate patients and their families about postoperative complications.

Clinical pathways for the treatment of cancer might prove difficult because of individual differences in both patients and surgeons ${ }^{15)}$. A balance between autonomy and standardization must be obtained ${ }^{8)}$. In the clinical setting, such a balance can lead to good management and hence enable medical staff to detect variations at an early stage, and therefore treat the individual appropriately. Thus, implementation of tailor-made clinical pathways may enable early detection and treatment for these variations.

In a strategy to reduce patient-related variance, we must adhere to guidelines for the prevention of SSI presented by the Centers for Disease Control ${ }^{23)}$. Early feeding ${ }^{24)}$ and sham feeding ${ }^{25)}$ also might decrease the rate of ileus. To decrease the rate of variance, we have devised four recommendations: implementing a clinical pathway for patients; informing patients and their relatives about potential complications; detecting variations at an early stage; and ensuring complications are managed efficiently according to individual clinical situations.

In summary, our results demonstrate that the postoperative risk predicted by POSSUM does not correlate with the observed morbidity rate. It is possible that it can predict a subset of patients with variance caused by complications, but due to its poor specificity we would not permit its clinical use. However, POSSUM may be a useful educational tool for informing patients and their relatives about potential postoperative complications during the clinical pathway process.

\section{References}

1) Nomura K : General rule of critical path for outcome management. J Jpn Soc Health Care Manage $4: 360-364$ (2003) (in Japanese)

2) Zander K : Use of variance from clinical paths: coming of age. Clin Perform Uual Health Care 5 : 20-30 (1997)

3) Jones HJS and de Cossart L : Risk scoring in surgical patients. Br J Surg 86 : 149-157 (1999) 
4) Copeland GP, Jones D and Walters M : POSSUM : a scoring system for surgical audit. Br J Surg 78 : 355-360 (1991)

5) Jones D, Copeland GP and de Cossart L: Comparison of POSSUM with APACHE II for prediction of outcome from a surgical high-dependency unit. Br J Surg 79 : 1293-1296 (1992)

6) Yasuda N, Watanabe M, Kusano T, Kamisaka K, Tsunoda A and Kusano M: The study on 6 goals by self-check in a clinical path for gastrointestinal cancers. Surgery $65: 461-463$ (2003) (in Japanese)

7) Yasuda N, Watanabe M, Kusano T, Tsunoda A and Kusano M: The study on clinical pathway with Six goals by self-check. J Jpn Coll Surg $28: 698-701$ (2003) (in Japanese)

8) Pearson SD, Goulart-Fisher D and Lee TH : Critical pathways as a strategy for improving care: problems and potential. Ann Intern Med 123 : 941-948 (1995)

9) Luttman RJ, Laffel GL and Peason SD: Using PERT/CPM (Program Evaluation and Review technique/ Critical Path Method) to design and improve clinical processes. Qual Manage Health Care 3 : 1-13 (1995)

10) D'Aquila NW : Facilitating inservice programs through PERT/CPM. Project Evaluation and Review Technique/Critical Path Method. Nurs Manage 24 : 92-94 (1993)

11) Coffey RJ, Richards JS, Remmert CS, LeRoy SS, Schoville RR and Baldwin PJ : An introduction to critical paths. Qual Manage Health Care 1 : 45-54 (1992)

12) Hofmann PA : Critical path method: an important tool for coordinating clinical care. Jt Comm J Qual Improv 19 : 235-246 (1993)

13) Spath PL: Critical paths: maximizing patients care coordination. Todays OR Nurse 17 : 13-20 (1995)

14) Gadacz TR, Adkins RB Jr and O'Leary JP: General surgical clinical pathways: an introduction. Am Surg 63 : 107-110 (1997)

15) Konishi T and Agawa S : Clinical pathways in Oncology. Jpn J Cancer Chemother 27: 655-670 (2000) (in Japanese)

16) Pritts TA, Nussbaum MS, Flesch LV, Fegelman EJ, Parikh AA and Fischer JE : Implementation of a clinical pathway decreases length of stay and cost for bowel resection. Ann Surg 230 : 728-733 (1999)

17) Stephen AE and Berger DL: Shortened length of stay and hospital cost reduction with implementation of an accelerated clinical care pathway after elective colon resection. Surgery $133: 277-282$ (2003)

18) Basse L, Jakobsen DH, Per Billesbolle RN, Werner $\mathbf{M}$ and Kehlet $\mathbf{H}$ : A clinical pathway to accelerate recovery after colonic resection. Ann Surg $232: 51-57$ (2000)

19) Haga Y, Ikei $S$ and Ogawa $M$ : Estimation of physiologic ability and surgical stress (E-PASS) as a new prediction scoring system for postoperative morbidity and mortality following elective gastrointestinal surgery. Surg Today 29 : 219-225 (1999).

20) Haga Y, Ikei S, Wada Y, Takeuchi H, Sameshima H, Kimura O and Furuya T: Evaluation of an estimation of physiologic ability and surgical stress (E-PASS) scoring system to predict postoperative risk: A multicenter prospective study. Surg Today $31: 569-574$ (2001)

21) Tekkis PP, Kessaris N, Kocher HM, Poloniecki JD, Lyttle J and Windsor CJ : Evaluation of POSSUM and P-POSSUM scoring system in patients undergoing colorectal surgery. Br J Surg 90:340-345 (2003)

22) Yii MK and $\mathrm{Ng} \mathrm{KJ}:$ Risk-adjusted surgical audit with the POSSUM scoring system in a developing country. Br J Surg 89 : 110-113 (2002)

23) Mangram AJ, Horan TC, Peatson ML, Silver LC and Jarvis WR: Guideline for prevention of surgical site infection, 1999. Am J Infect Control $27: 97-132$ (1999)

24) DiFronzo LA, Cymerman J and O'Connell TX. Factors affecting early postoperative feeding following elective open colon resection. Arch Surg 134 : 941-946 (1999)

25) Asao T, Kuwano H, Nakamura J, Morinaga N, Hirayama I and Ide M: Gum chewing enhances early recovery from postoperative ileus after laparoscopic colectomy. J Am Coll Surg 195:30-32 (2002)

[Received August 20, 2004 : Accepted December 17, 2004] 